\title{
Design and Construction of High-speed Dynamic Balance for High Load
}

\author{
Junli Sun ${ }^{\mathrm{a}}$, Nong Chen and Jin Liu \\ China Academy of Aerospace Aerodynamics, Beijing 100074, P.R. China
}

\begin{abstract}
There are high longitudinal loads and mismatched longitudinal/lateral aerodynamic characteristics of unconventional aerodynamic configurations, which present a new challenge to high-speed dynamic balance technology. The wind tunnel dimension limitation makes a new command in miniaturization design of dynamic balance. The article describes a solution method which is based on the electromechanical motion modulation testing principle and mechanical structure of saddle-type dynamic balance. There is an II-type structure assembled by oil film bearing and elastic pivot beam on the outer tube to achieve motion modulation and measurement. The double variable cross-section vertical beams are placed in front of inner shaft to measure the five components of high load. The double saddle-type structure is applied to connect the intermediate part of inner shaft with outer tube together. A Scotch yoke mechanism is composed of the transmission eccentric cam of sting and a mullion structure which is designed at the rear part of inner shaft. According to the redesign and alteration, the effective lateral load signal output could be obtained from the miniaturized dynamic balance, in which the maximum longitudinal load bearing capacity is extended more than twice. A great agreement is also obtained between the measurement accuracy and design requirements.
\end{abstract}

\section{Introduction}

The unconventional configuration design requirements are increased and the flight envelopes are expended gradually with the development of aerospace aircraft. It becomes much more serious about the unsteady and asymmetrical separation of the flow around aircraft, which raises a crucial point to make accurate prediction about characterizes of unsteady and dynamic [1-4]. The dynamic stability technology which based on the weak signal detection technique is a solution often used in aerodynamics. The direct, cross and cross-couple dynamic derivative parameters on three degrees of freedom of pitch, yaw and roll are obtained by motion modulation of the aircraft. The dynamic balance is a kernel part in this special experiment technology. The dynamic balance could achieve the motion modulation and measurement by its high flexibility, and its high stiffness ensures to measure aerodynamic force response and avoid interference of noise, while the disadvantage is the limitation of dimension and aerodynamic loads. The high longitudinal loads and mismatched longitudinal/lateral aerodynamic characteristics of unconventional aerodynamic configuration aircrafts lead to the redesign and alteration of dynamic balance.

The international researchers mainly adopt split shape and integrated shape of high-speed wind

a Corresponding author: sunjunli@zju.edu.cn 
tunnel dynamic balances structure to achieve the two major functions: motion modulation and aerodynamic forces response measurement [5-15]. The split shape is front-rear series structure, whose front part often adopt the high stiffness conventional five-component balance, and the rear part is composed of an elastic bearing / ball bearing acted as vibration center and a Scotch yoke mechanism which is a reciprocating motion mechanism to converting rotational motion into linear motion [5-9], which results to high axial dimension and space occupation, so that it is suitable for high slenderness ratio aircraft. The dynamic balance suffers a large inertial force by the large space among calibration center, model gravity center and vibration center, which leads to the limitation on sensitivity level of torque components. It raises the difficulty of the weak physical parameters measurement $[5,6,10,11]$. The low signal-to-noise ratio and the weak suppression ability on noise decrease the test accuracy. Particularly, some unreasonable design of split shape dynamic balances lead to the resonance with wind tunnel sting, which will destroy the wake of aircraft and mistake measurement results. Two kinds of integrated shapes are in common use of dynamic test. One of them is a saddle-type structure. The inner shaft is designed as a ball bearing to associate with longitudinal moment beam to achieve motion modulation and measurement. The front part of outer shaft is designed as a rectangle beam to measure the five components. The immediate part of outer shaft which sits at the vibration center of inner shaft structure seems like a saddle structure. The rear part is a mullion structure which makes up a Scotch yoke mechanics with the transmission eccentric cam of wind tunnel sting. The front, immediate and rear part of outer shaft are assembled together before the assembly with inner shaft $[10,11,12]$. The whole structure is compactness and the space is much tinier among calibration center, model gravity center and vibration center. For the reason of assembled structure, it is hard to overcome the disadvantages of assembled structure, such as creep deformation, internal friction, hysteresis, non-liner interference and so on. The other one is a can-style structure. Its inner shaft is composed of cross-type elastic bearing used as a vibration center and the moment beam attached at the transmission mechanic in the wind tunnel sting. The outer structure is designed to be a wheel hub structure to obtain the five components $[13,14]$, or vice versa [15]. The limited carrying capacity exists in some parts of the dynamic balance, like the inner shaft elastic bearing, torque beam and wheel hub structure of outer shaft which could not support the high aerodynamic loads. Furthermore, the integrated shape structure has higher measurement accuracy, while the radial dimension limitation of aircraft model allows it only fit for the 2 meters degree wind tunnel or larger. The split shape dynamic balance does not own high measurement accuracy, which is allowed to be used in the 2 meters degree wind tunnel or less for the reason of the axis dimension limitation of aircraft model, even just in high slenderness ratio aircraft.

The unconventional aerodynamic configuration aircraft is being focused in the article. For example, the normal aerodynamic force of flying-wing aircraft increases apparently with the raise of angle of attack, even reaches to 1000 kilograms $\left(\mathrm{Ma}=0.9, \alpha=28^{\circ}\right)$ and the longitudinal/lateral aerodynamic characteristics are extremely mismatched (ratio of longitudinal load to lateral load equals to 20:1), which could bring measurement problems in wind tunnel experiment about the unsteady and dynamic characterizes [16,17]. It is necessary to redesign the split shape dynamic balances to satisfied the measurement accuracy requirements of dynamic characterizes [3,9]. In addition, the miniaturization design should be implemented for the reason that the test section dimension limitation of 1.2 meters degree wind tunnel makes a large constraint to the dynamic balance. The article is based on the saddle-type structure in order to solve the problems above. There is an II-type beam structure assembled by oil film bearing and elastic pivot on the outer tube to achieve motion modulation and measurement. The double variable cross-section vertical beams are placed in front of inner shaft to measure the five components of high loads. The double saddle-type structure is applied to connect the intermediate part of inner shaft with outer tube together. The inner shaft and outer tube could be welded together through the oil film bearing by the electron beam, and elastic pivot could be replaced according to the aerodynamic trim torque. A Scotch yoke mechanism is composed of the transmission eccentric cam of sting and a mullion structure which is designed at rear part of inner shaft. According to the redesign and alteration, the sensitivity of lateral load could be improved to achieve the five-component sensitivity measurement requirements from the miniaturized dynamic balance, in which the maximum longitudinal load bearing capacity is extended more than twice. The measurement accuracy could reach the design requirements. 


\section{Basic principles of the apparatus}

The flow structure changed rapidly along with the raise of angle of attack and aerodynamic loads present the obvious unsteady features during the flight. The physical variables, like pressure, velocity, density, have changed with time since the interaction of various physical phenomena such as flow separation, shock wave boundary layer interference. The unsteady aerodynamic loads are much smaller than the static aerodynamic loads. Since most of the aerodynamic load signals are slowly varying over time or are approximated of a non-time-dependent direct-current (DC) component, which means the static aerodynamic load component is distributed in the low frequency region of signal. Suppose a conventional DC-type amplifier (such as a conventional balance) is used to amplify and detect a weak unsteady aerodynamic load signal directly, the $1 / \mathrm{f}$ noise and drift will be existed with seriously influence on the detection sensitivity.

The unsteady aerodynamic load measurement should adopt the modulation frequency technology to translate the signal in the frequency axis to avoid the $1 / \mathrm{f}$ noise and DC drift, which also ensures to keep the Dynamic Similarity Criteria between the dynamic frequency of the aircraft and its wind tunnel model, which is conducive to the unsteady aerodynamic load detection. It is necessary to suppress the noise by increasing the amplitude in the low test frequency (schematic diagram shown in figure 1) to improve signal-to-noise ratio. A modulation amplitude technique is required when the noise power spectrum of an unsteady aerodynamic load signal approaches to a low frequency. In general, the amplitude in low-speed wind tunnels is much larger than high-speed wind tunnel dynamic tests.

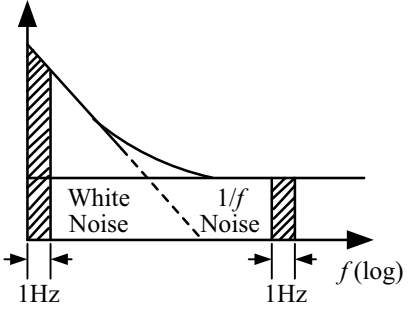

Figure 1. Amplitude modulation of the signal can reduce the output noise.

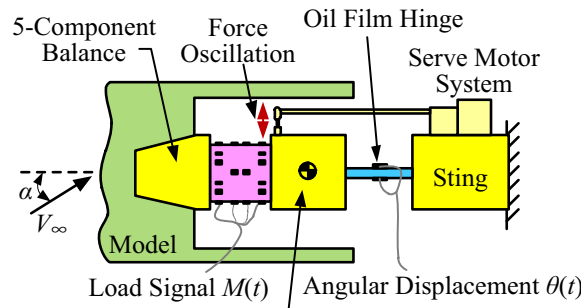

Figure 2. Schematic diagram of dynamic balance mechanical mechanism.

Figure 2 presents a schematic diagram about the electromechanical motion modulation testing principle and the mechanical structure of the saddle-type dynamic balance. Taking pitch/yaw dynamic balance as an example, the maximum frequency of the aerodynamic load signal $f(x)$ of the dynamic balance is assumed to $\omega_{0}$ and the rotary switch modulation shown in figure 3 is adopted, a reasonable switch modulation function $S_{k}(t)$ shown in figure 4 is designed to simulate the pitching motion of the aircraft in figure 5. The output of the modulator (i.e. the output of the dynamic balance five-component) is the convolution of aerodynamic load signal $f(x)$ with switching function $S_{k}(t)$.

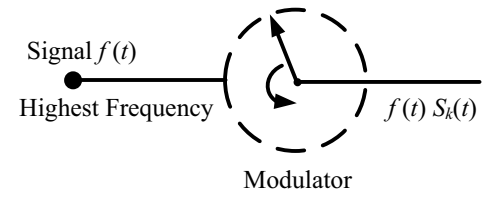

Figure 3. Pneumatic load signal modulation.

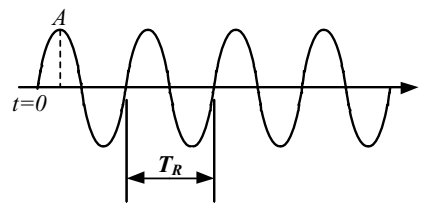

Figure 4. Switch modulation function $S_{k}(\mathrm{t})$.

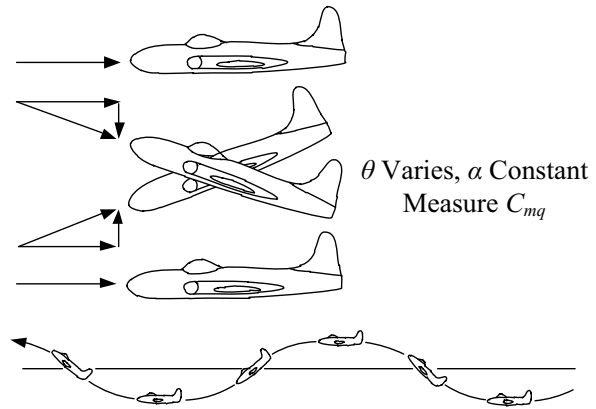

Figure 5. Aircraft pitching motion diagram. 
The spectrum of the aerodynamic load signal $f(x)$ is represented by the Fourier transform $F(t)$. The modulated aerodynamic load signal could be expressed as $X(t)=f(x) * S_{k}(t)$, and its spectrum can also be represented by the Fourier Transform $X(f)$ :

$$
X(f)=\int_{-\infty}^{\infty} S_{k}(t) f(t) e^{-j \omega t} d t
$$

The switch modulation function $S_{k}(t)$ is accomplished by next expressions:

$$
S_{k}(t)=A e^{j \omega_{R} t}
$$

Where:

$\omega_{R}=2 \pi / T_{R}$ is the fundamental frequency of the modulator. A, TR are the amplitude and period of the switching modulation function shown in figure 4.

From the formula (1) and formula (2), it could form:

$$
S_{k}(t) f(t)=f(t) \cdot A e^{j \omega_{R} t}
$$

And

$$
X(f)=\int_{-\infty}^{\infty} f(t) \cdot A e^{-j\left(\omega-\omega_{R}\right) t} d t
$$

Due to the Fourier transform of the function $f(t)$, it could obtains:

$$
F(f)=\int_{-\infty}^{\infty} f(t) e^{-j \omega t} d t
$$

The equation (5) could be written as:

$$
F\left(f-n f_{R}\right)=\int_{-\infty}^{\infty} f(t) e^{-j\left(\omega-\omega_{R}\right) t} d t
$$

Substituting equation (6) into equation (4):

$$
X(f)=A \cdot F\left(f-n f_{R}\right)
$$

Getting the spectrum of signal after modulation, the discussion of frequency spectrum is as follows: For $n=0$, where

$$
X(f)=A \cdot F(f)
$$

For $n=i$, where

$$
X(f)=A \cdot F\left(f-i f_{R}\right)
$$

The corresponding demodulation algorithm [2, 3, 5-16] could be used to restore the original aerodynamic load signal $f(t)$ from the modulated aerodynamic load signal $X(t)$.

The weak signal detection technology based on the principle above is different from the general detection technology. The weak signal detection focuses on how to suppress noise and improve the signal-to-noise ratio rather than the physical model, sensing principle, the signal conversion circuit and instrument implementation. The dynamic balance is the special electromechanical motion modulation sensor which has been applied of weak signal detection technology. It could achieve the motion modulation and measurement by high flexibility, measure aerodynamic force response and avoid extraneous effects by high stiffness. 


\section{Apparatus description}

\subsection{Design loads and condition}

The wind tunnel experiment is to be carried out in the wind tunnel of 1.2 meters. The internal space dimension is $\Phi 55 \mathrm{~mm}$ (maximum diameter) $\times 280 \mathrm{~mm}$ (axial distance) for the flying wing model. According to the static test results [16], the lateral load of the dynamic balance is enlarged by considering the experimental scheme, and the design load of the dynamic balance is determined as shown in Table 1.

Table 1. The design scale and precision of the dynamic balance.

\begin{tabular}{cccc}
\hline Load Component & Range $(\mathrm{N}, \mathrm{Nm})$ & Accuracy $(\%)$ & Precision (\%) \\
\hline$Y$ & 10000 & $\leq 0.5$ & $\leq 0.2$ \\
$Z$ & 300 & $\leq 0.5$ & $\leq 0.2$ \\
$M_{x}$ & 30 & $\leq 0.5$ & $\leq 0.2$ \\
$M_{y}$ & 18 & $\leq 0.5$ & $\leq 0.2$ \\
$M_{z}$ & 80 & $\leq 0.5$ & $\leq 0.2$ \\
\hline
\end{tabular}

\subsection{Whole structure of high-load dynamic balance}

The developed dynamic balance based on the saddle-type dynamic balance structure is constructed of inner shaft and outer concentric tube, as shown in figure 6.

First of all, the inner shaft is machined strain-gauged beams for force measurement, this includes: the double variable cross-section vertical beam structure with the 1:10 conical surface section in front of the inner shaft which were used to measure five-component force and attach the model, the inner shaft and the outer tube attached at the double saddle-type beams of the middle part, which to make inner shaft become the 'force-floating' section of outer tube. A horizontal mullion structure placed at rear of inner shaft which can be combined with the eccentric cam in wind tunnel support sting to form a Scotch yoke mechanism.

The double variable cross-section vertical beam structure is regarded as strain-gauged section design. It overcomes the defects of sheet-beam structure which is good at anti-pitching torque but weak in normal stiffness, and solves the problem of column-beam structure which has large stiffness and high sensitivity but difficulty to match longitudinal and lateral loads. It is great in longitudinal load bearing capacity and lateral sensitivity output level for the new strain-gauged section.

Secondly, the outer tube is mainly used to achieve motion modulation and measurement. The front section of the outer tube attached with inner shaft is constructed of "oil film bearing+ elastic pivot" II-type beams structure. The front "I"-type structure is a fixed oil film bearing, through which the inner and outer shaft structure can be welded by electron beam, so that it can allow the rotation of inner shaft around the axis of oil film bearing. The rear "I" -type structure is a detachable elastic pivot, on which the strain gauges are attached. The elastic pivot can be replaced according to the aerodynamic trim torque. The rear of the outer tube is 1:10 conical surface section which is applied to connect the wind tunnel sting.

The role of the double-beam structure is similar to elastic bearing of existed dynamic balance [3,9], the oil film bearing adopted by the front "I"-type structure could enhance the carrying capacity and effectively reduce the mechanical damping. The rear "I"-type structure can output the pitch motion modulation signal in real time for data reducing. Besides, the double-beam structure acted as a connection structure reduces the distance to $35 \mathrm{~mm}$ among the vibration center of developed dynamic balance, model center of mass and the calibration center. Compared with the existing dynamic balance $[3,9]$, the developed dynamic balance effectively shortens the distance between the vibration center and the calibration center, greatly reduces the axial length of the size and improves the measurement accuracy. 


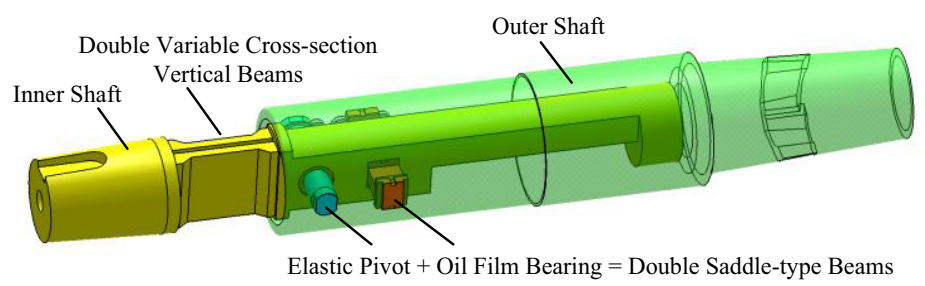

Figure 6. Structure of dynamic balance.

In summary, the saddle-type dynamic balance based on the double variable cross-section vertical beam / double saddle-type beams structure makes the high-load saddle-type dynamic balance compact and miniaturized. The high stiffness of the double-variable cross-section vertical beams structure not only can withstand the high load and model gravity, even can reduce the impact of noise, but also to ensure the lateral output magnitude. The double saddle-type beams structure which could solve the bearing problem of the motion modulation mechanism, but also effectively trim the aerodynamic torque and record motion modulated signal. The whole device owns higher measurement accuracy than before.

\subsection{Miniaturization optimization design of the whole structure}

The problems of high longitudinal load and extreme mismatch of longitudinal/lateral aerodynamic forces are solved by the double-variable cross-section vertical beam / double saddle-type beams structure. Due to the limitation of the wind tunnel test section and the inner space of aircraft test model, the entire dynamic balance needs to be constrained in the limited space.

The conical surface and the mullion structure of the inner shaft play the connection and motion transmission function. These parts are adopted by the high stiffness structure which will not affect the motion modulation and aerodynamic measurement function for its small deformation. The double variable cross-section vertical beam / double saddle-type beams structure are the main bearing and measuring structure, which should be optimized in necessary. In addition, the little influence of the high-stiffness structure leads to its omission in the optimization model. The optimization model should be preciseness and effective to avoid the redundant calculation.

In summary, The optimization design of the dynamic balance can be simplified as a double variable cross-section vertical beam / double saddle-type beams structure shown in figure 7 .

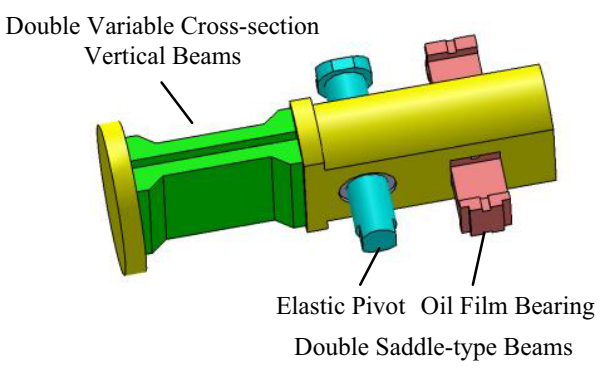

Figure 7. Double variable cross-section vertical beam / double saddle-type beams structure.

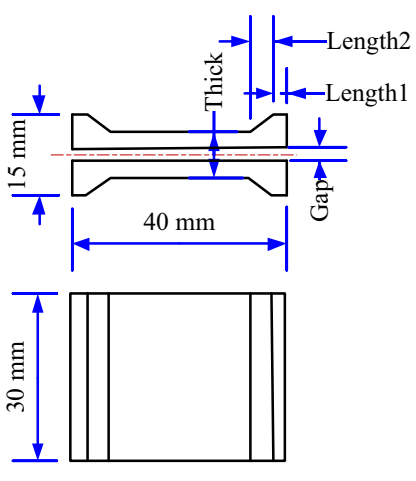

Figure 8. Dimension parameters of double variable cross-section vertical beam.

According to experiment requirements and design experience, the dynamic balance can be further optimized on the dimension of the cross section vertical beam as shown in figure 8 .

Four typical results of ten outputs are chosen for processing after the five components load simulation, which include: the stress under the longitudinal force and torque applied alone, the strain under the lateral force and torque applied alone. The optimization target requires to lower stress under 
the longitudinal force and to increase the lateral load strain. Thus, the mathematical model of dynamic balance optimization is:

$$
\begin{aligned}
& \min \mathrm{Y} 10000 \mathrm{~S}(\boldsymbol{x}), \operatorname{Mz} 600 \mathrm{~S}(\boldsymbol{x}) \\
& \max \quad \mathrm{Z} 800 \mathrm{E}(\boldsymbol{x}), \operatorname{My} 50 \mathrm{E}(\boldsymbol{x}) \\
& \text { s.t. }\left\{\begin{array}{l}
0.6 \leq \text { Gap } \leq 3.0 \\
1.0 \leq \text { Length } 2 \leq 5.0 \\
1.0 \leq \text { Length } 1 \leq 5.0 \\
8.0 \leq \text { Thick } \leq 12.0
\end{array}\right.
\end{aligned}
$$

Where:

Y10000S $(x)$ means the relationship between value $x$ and stress under normal force $\mathrm{Y}=10000 \mathrm{~N}$;

$\operatorname{Mz} 600 \mathrm{~S}(x)$ means the relationship between value $x$ and stress under normal torque $\mathrm{Mz}=600 \mathrm{Nm}$;

$\mathrm{Z} 800 \mathrm{E}(x)$ means the relationship between value $x$ and strain under lateral force $\mathrm{Z}=800 \mathrm{~N}$;

$\operatorname{My} 500 \mathrm{E}(x)$ means the relationship between value $x$ and strain under lateral torque $\mathrm{My}=50 \mathrm{Nm}$;

Value $x$ could be written as a matrix: $\boldsymbol{x}=\left[\begin{array}{llll}x(1) & x(2) & x(3) & x(4)\end{array}\right]=[$ Gap Length2 Length1 Thick].

The optimization method is based on the multi-objective optimization algorithm, and Design of Experiment (DOE) method is used to find the most suitable parameters as the initial values. Finally, the optimized Pareto set would be output after implementing an optimization algorithm for a plurality of sub-targets. The parameters of optimized dynamic balance also have been determined by considering the processing capacity. The length of the dynamic balance is $335 \mathrm{~mm}$, of which the outer diameter is $50 \mathrm{~mm}$ and the inner diameter is $36 \mathrm{~mm}$. Among the parameters of double variable cross-section vertical beam, the parameter named Length 1 is $3 \mathrm{~mm}$, Length 2 is $4 \mathrm{~mm}$, the Thick is $9 \mathrm{~mm}$ and Gap is $2 \mathrm{~mm}$.

\section{Calibration results}

The general static/dynamic calibration on dynamic balance could adopt two kinds of methods: the ground test and the numerical simulation test. The purpose of the static calibration is similar to the conventional static balance calibration, while dynamic calibration aims to detect the frequency modulation and amplitude modulation capability of the motion modulation mechanism as well as the dynamic response characteristics of the input / output of the dynamic balance system. In this paper, numerical method is used to calibrate the developed dynamic balance.

\subsection{Finite element model of whole structure}

The appropriate simplification has been done on the whole structure to facilitate the meshing and numerical calculation by omitting keyway, wedge hole and other irrelevant parts. The global meshing uses a quadratic tetrahedron mesh. The local grid refinement method is applied on the strain-gauged section and the double-variable cross-section vertical beam / double saddle-type beams structure. The total number of grid cells is 422167 and the total number of nodes is 641882 , as shown in figure 9 .

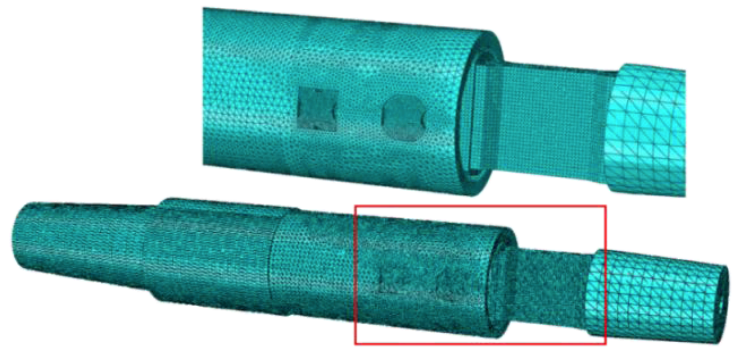

Figure 9. Finite element model of whole structure. 
F141 (00Ni18Mo5Co9TiAl) is chosen as the material of dynamic balance, whose Young modulus is $1870 \mathrm{MPa}$ and the yield limit is $1754 \mathrm{MPa}$. The safety factor is taken as $n=1.5$ so that the available stress $[\sigma]$ is equal to $1170 \mathrm{MPa}$. The details about the F141 are shown in table 2.

Table 2. Mechanical properties of material $\left(20^{\circ} \mathrm{C}\right)$.

\begin{tabular}{cccccc}
\hline Material & $\rho\left(\mathrm{kgm}^{-3}\right)$ & $E\left(\mathrm{Nm}^{2}\right)$ & $\mu$ & $\sigma_{s}(\mathrm{MPa})$ & $\sigma_{b}(\mathrm{MPa})$ \\
\hline F141 & 7800 & $1.87 \mathrm{e} 11$ & 0.29 & 1754 & 1862 \\
\hline
\end{tabular}

The fixed-boundary condition is adopted on the taper section of outer tube of the dynamic balance, and the binding constraints are made between the remaining parts. Load condition is considered as two situations: static calibration and dynamic calibration.

\subsection{The static calibration}

The whole finite element model is established as a linear model which is in accordance with the test requirements. In static analysis, linear change in the static load will produce a linear change in the results during the static analysis. The load case on the dynamic balance would only be considered in a single direction. The single-component loading results are shown in table 3.

Table 3. Single-component finite element simulation results on dynamic balance.

\begin{tabular}{ccccc}
\hline Load Component & Value $(\mathrm{N}, \mathrm{Nm})$ & $\sigma_{\max }(\mathrm{Pa})$ & $\varepsilon_{\max }$ & $U(\mathrm{~m})$ \\
\hline$Y$ & 10000 & $1.944 \mathrm{e} 8$ & $1.057 \mathrm{e}-3$ & $1.219 \mathrm{e}-4$ \\
$Z$ & 300 & $2.631 \mathrm{e} 7$ & $1.479 \mathrm{e}-4$ & $2.699 \mathrm{e}-5$ \\
$M_{x}$ & 30 & $1.505 \mathrm{e} 8$ & $8.270 \mathrm{e}-4$ & $1.358 \mathrm{e}-4$ \\
$M_{y}$ & 18 & $4.653 \mathrm{e} 7$ & $2.585 \mathrm{e}-4$ & $1.551 \mathrm{e}-4$ \\
$M_{z}$ & 80 & $8.817 \mathrm{e} 7$ & $4.752 \mathrm{e}-4$ & $1.094 \mathrm{e}-4$ \\
\hline
\end{tabular}

The strain-gauged section of dynamic balance could meet the design requirements with a bit lower about lateral output. However, the distance between the aerodynamic rotation center and the geometric center is equivalent to applying an extra moment on the dynamic balance after transferring the load from geometric center to rotation center and resulting to enhance the effect of the lateral load.

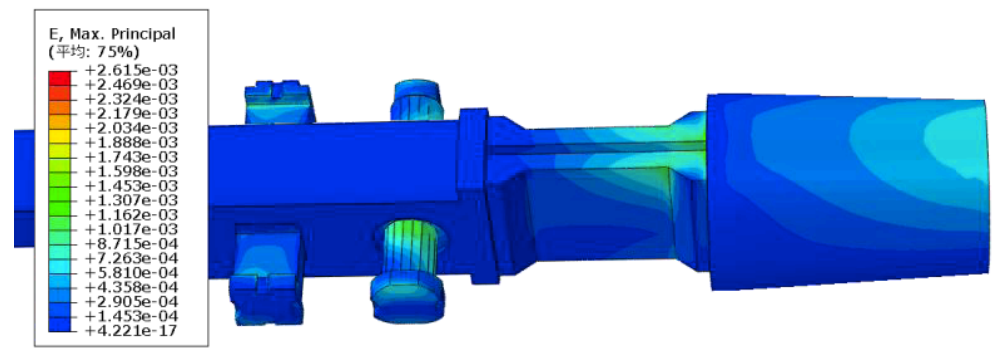

Figure10. Local strain cloud diagram of dynamic balance at $\mathrm{Ma}=0.8$ and $\alpha=12^{\circ}$.

The local strain cloud diagram in figure 10 visually shows that the root on strain-gauged section is the location where the stress concentration occurs, and the stress gradient is large with the strain increases sharply. It is necessary to ensure the stress concentration region is within allowable stress $[\sigma]$. In addition, the elastic pivot fixed together on the outer tube of dynamic balance, whose junction part is also a weak point, but the strain value and gradient are smaller to the root on strain-gauged section.

The stress and strain distributions under the wind tunnel load are plotted in figure 11 by selecting a characteristic edge on the top surface of the strain-gauged section. The effect of wind tunnel load is more prominent than the design load to meet the strain signal output requirements. Besides, the stress gradient on both sides of the curve increases rapidly, which indicates the stress concentration range at the root of the strain-gauged section and becomes more prominent at the high angle of attack. 


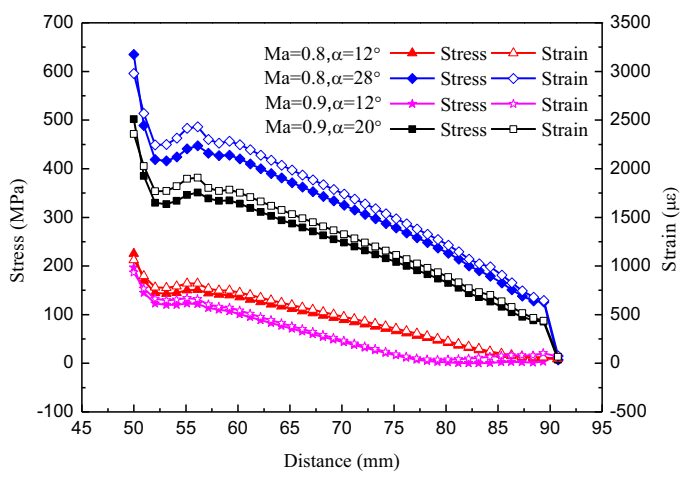

Figure 11. Stress/strain distribution on the upper surface of the strain-gauged section.

The main reason for the high $\sigma_{\max }$ is the stress concentration at the root of strain-gauged section and the strain gauge area can guarantee the effective output without any damage. The high $\sigma_{\max }$ exceeded the allowable stress $[\sigma]$ at $\mathrm{Ma}=0.8$ and $\alpha=28^{\circ}$ does not reach to the yield limit of the material. Moreover, the impact load to the dynamic balance is small which will not beyond the material limitation from $\alpha=0{ }^{\circ}$ to $\alpha=28^{\circ}$ after the wind tunnel uniform flow field has been established.

\subsection{The dynamic calibration}

The dynamic analysis of the dynamic balance contains many aspects: the modal analysis, harmonic response analysis, spectrum analysis and other dynamic analysis.

Finite element modal analysis shows the natural frequency of dynamic balance is very high which would not reach the mechanical system resonance to cause the structural damage.

The displacement function $x=2 \times 10^{-3} \sin (10 \pi \mathrm{t})$ is imported into the dynamic balance to simulate the forced vibration under finite element harmonic response analysis. The displacement-time curves shown in figure 12 are drawn from the points on the strain-gauged section and the oil film bearing.

A nice agreement is obtained between the output and given-frequency of the dynamic balance structure system. The response frequency is approximately $5 \mathrm{~Hz}$ which is close to the input frequency. The structure can effectively reproduce the forced vibration of $\pm 1^{\circ}$ by the response results. A few attenuated occurs at the angular amplitude during the transmission while the effect is very small so that the forced vibration response can receive high precision. The voltage signal output of strain gauges on the oil film bearing could reach $500 \mathrm{mV} / \mathrm{V}$ which can make strain gauge work in normal. The voltage signal output of stain gauges on the strain-gauged section is very low due to lack of aerodynamic load. Actually, the output signal on the strain-gauged section can meet the working requirement under wind tunnel loads, which has been verified in the static calibration.
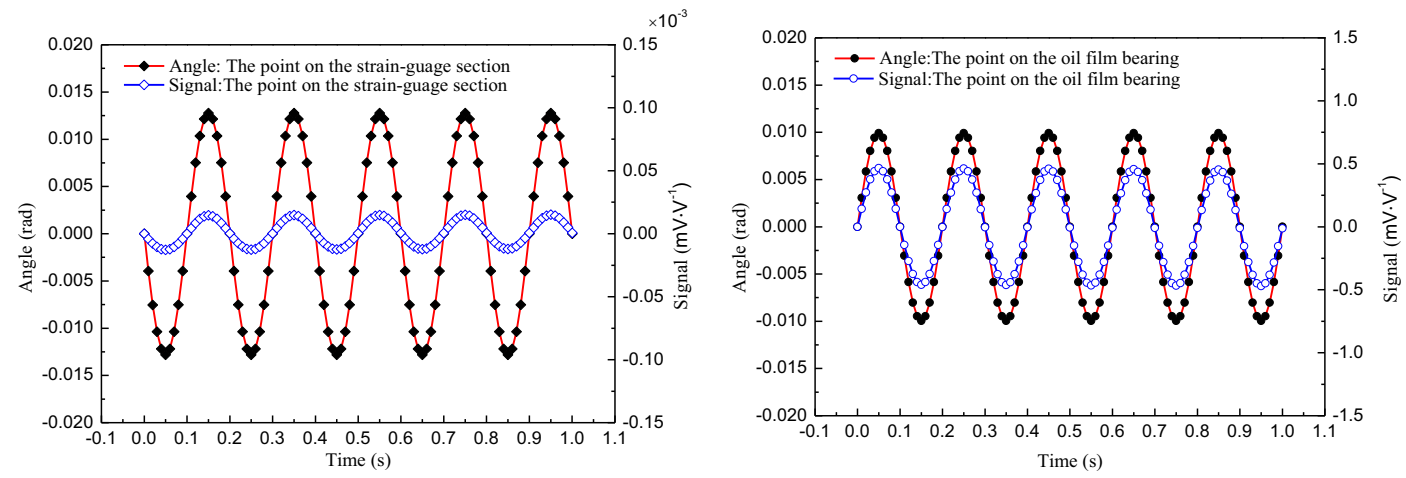

Figure 12. Displacement and output signals over time (Left: strain-gauged section, Right: oil film bearing). 
Therefore, the dynamic balance of static/dynamic calibration results has reached the design requirements. It could be considered that the dynamic balance would work normally to meet the security requirements and to obtain the high test accuracy.

\section{Conclusions}

The wind tunnel dynamic test measurement problems brought from the dynamic characteristics of unconventional configuration aircraft result to the developed dynamic balance integrated with the double-variable cross-section vertical beam / double saddle-type beams structure. The working principle, mechanical structure principle and whole structural design are expounded to the dynamic balance, which is regarded as a special kind of electromechanical motion modulation sensor. The miniaturization about the optimization design has been done based on the whole structural characteristics of the finite element model analysis, which reduces the radial dimension of the dynamic balance effectively. A complete design method could be established from the structure, optimization and finite element analysis.

According to the numerical simulation about the calibration on static/dynamic characterizes of the dynamic balance, the bearing capacity of two key substructures: motion modulation and aerodynamic response measurement are enhanced, whose maximum longitudinal load bearing capacity is extended more than twice. The sensitivity of lateral load is improved and a great agreement could be obtained between the measurement accuracy and design requirements. Such dynamic balance could be widely applied to high-load wind tunnel test, which owns good practicability and popularization value.

\section{References}

1. K.J. Orlikruckemann, NASA-CR-114583 (1973)

2. D.M.Tomek, W.G.Sewall, S.E.Mason, AIAA 2006-3148 (2006)

3. J Liu. Master Degree Thesis (2014)

4. J.M. Luckring, O.J. Boelens, AIAA 2015-0061 (2005)

5. C.J. Schueler, L.K. Ward, Hodapp. Jr. A.E, AD-669227 (1968)

6. C Wang, Acta Aerodynamic Sinica 5(2) (1987)

7. D He, X Gu, First International Symposium on Strain Gauge Balances (1999)

8. Makoto Ueno, Hitoshi Miwa, AIAA 2001-0406 (2001)

9. J Lin, N Chen, Y Song, J Hu, K Xie, APISAT-2014 (2014)

10. R.A. Kilgore, B.T. Averett, Journal of Aircraft 1(5) (1964)

11. K.J. Orlikruckemann, J.G. Laberge, E.S. Hanff, AIAA 74-611 (1974)

12. J.C. Uselton, B.L. Uselton, AIAA 75-211 (1975)

13. S.M. Coulter, T.D. Buchanan, AIAA 80-0451 (1980)

14. D.R. Haberman, AIAA 82-0595 (1982)

15. M. Samardzic, Z. Anastasijevic , D. Marinkovski, Proc. IMechE Part G: J. Aerospace Engineering, 226(11) (2011)

16. Y Li, H Liu, Y Huang, Acta Aerodynamic Sinica 34(1) (2016)

17. D.D. Vicroy, T.D. Loeser, Schuette Andreas, AIAA 2010-4394 (2010) 